\title{
Remnant-like Particle Cholesterol Measurement
}

National Cancer Institute

\section{Source}

National Cancer Institute. Remnant-like Particle Cholesterol Measurement. NCI

Thesaurus. Code C120655.

The determination of the RLP cholesterol present in a sample. 